\title{
Tetra-Rhenium Molecular Rectangles as Organizational Motifs for the Investigation of Ligand-Centered Mixed-Valency: Three Examples of Full Delocalization
}

\author{
Peter H. Dinolfo and Joseph T. Hupp* \\ Department of Chemistry \\ 2145 Sheridan Road, Northwestern University, Evanston, IL 60208-3113,USA
}

Supporting Information

Email: jthupp@chem.northwestern.edu

Submitted to J. Am. Chem. Soc. on 7/28/2004, revised 10/04/2004. 

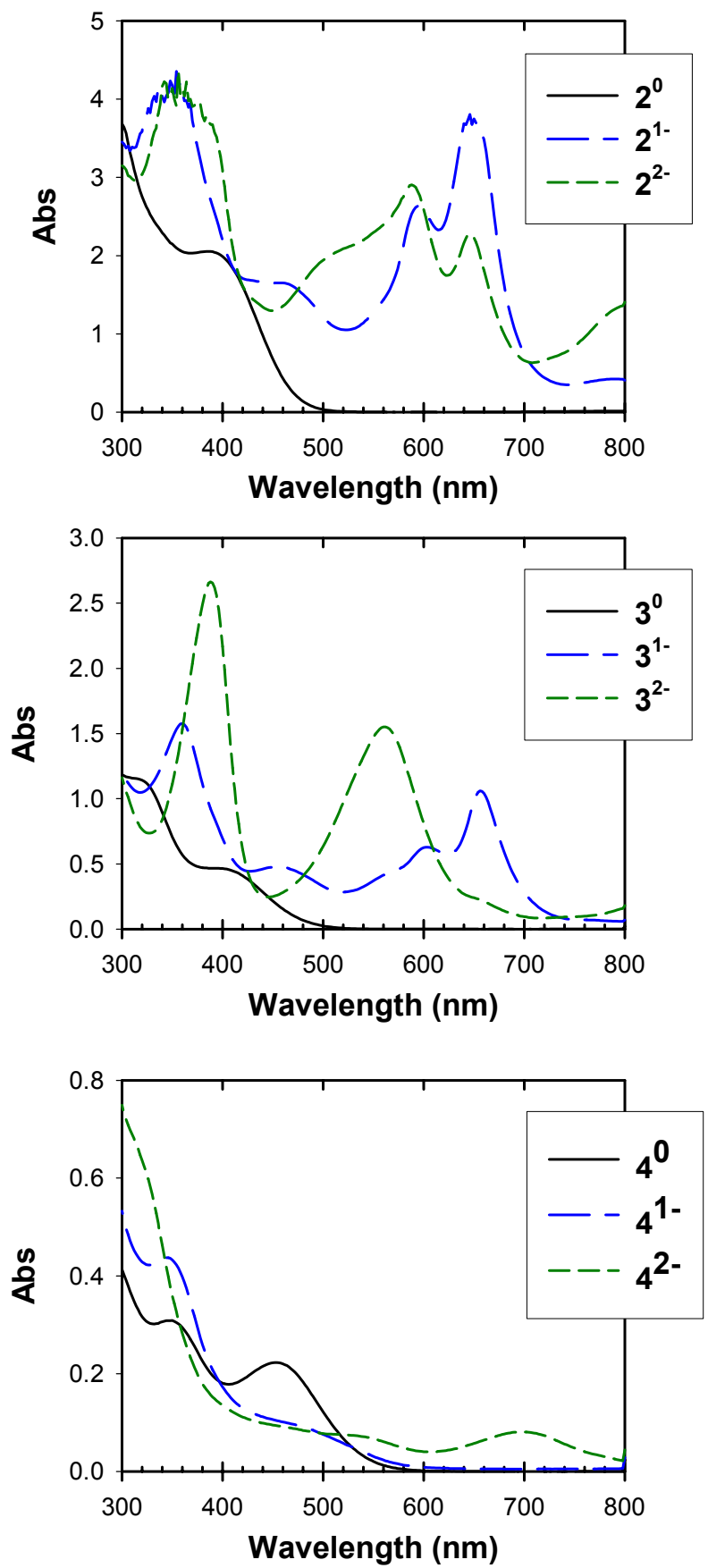

Figure S3. UV-Visible absorption data for the neutral (solid black line), 1- (long dashed green line) and 2- (short dashed blue line) states for $\mathbf{2}$ - $\mathbf{4}$ taken in THF, with $0.02 \mathrm{M}\left[\mathrm{NBu}_{4}\right]\left[\mathrm{B}\left(\mathrm{C}_{6} \mathrm{~F}_{5}\right)_{4}\right]$ as the supporting electrolyte. 


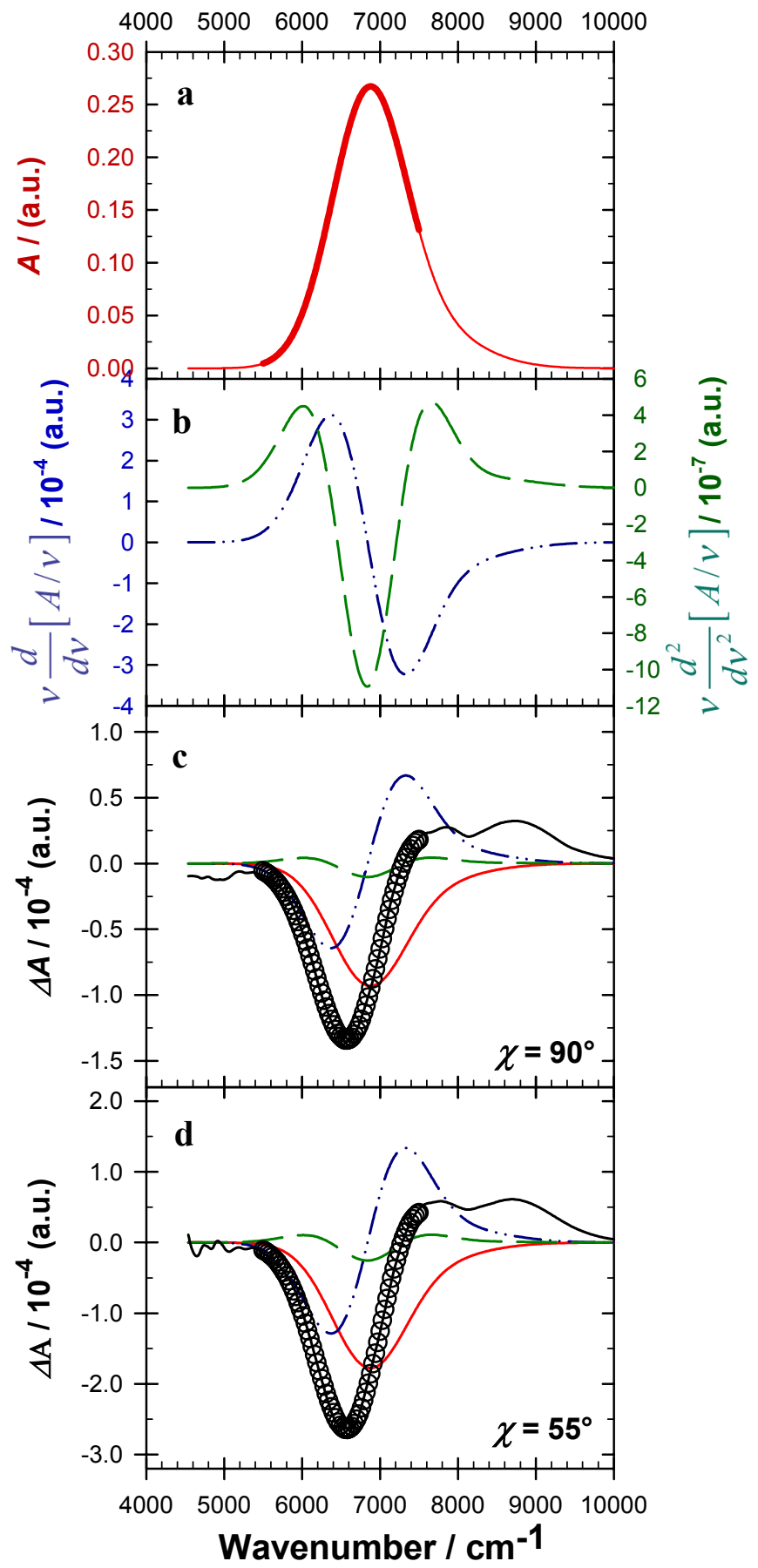

Figure S2. Liptay analysis of the Stark spectra for $\mathbf{2}^{1-}$. Panel a shows the unperturbed absorption specta at $77 \mathrm{~K}^{\circ}$ in butyronitrile (solid red line) and panel $\mathrm{b}$ shows the energy weighted first and second derivatives (dashed-dotted blue line and dashed green line respectively). Panels c and $\mathrm{d}$ show the measured (solid black lines) and fit (open circles) Stark signals at $\chi=90^{\circ}$ and $55^{\circ}$ respectively. Included with the fits are the contributions from the absorption spectra (red) and the first and second derivatives (dashed-dotted blue line and dashed green line respectively). 


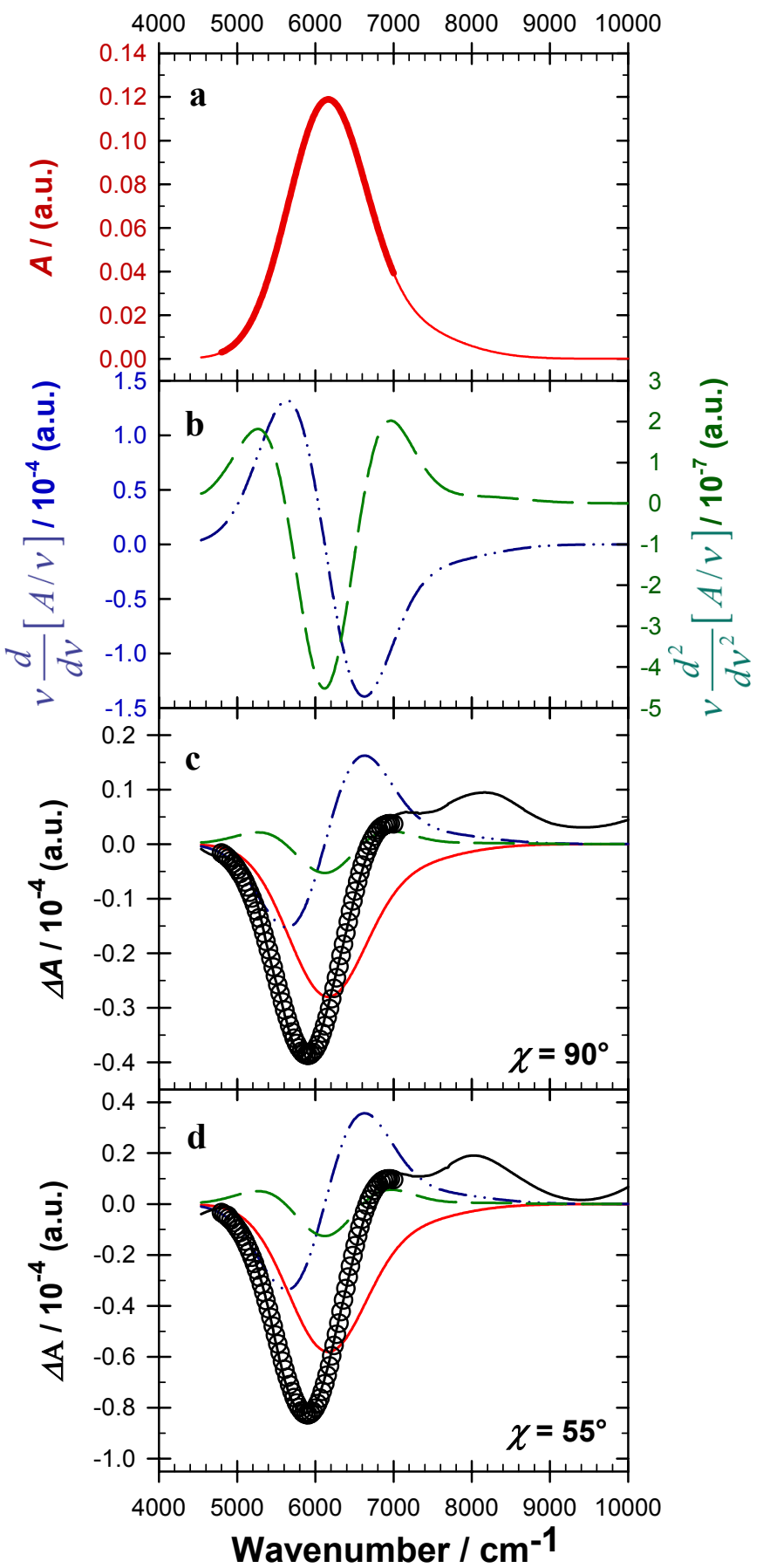

Figure S3. Liptay analysis of the Stark spectra for $\mathbf{3}^{1-}$. Panel a shows the unperturbed absorption specta at $77 \mathrm{~K}^{\circ}$ in butyronitrile (solid red line) and panel $b$ shows the energy weighted first and second derivatives (dashed-dotted blue line and dashed green line respectively). Panels c and $\mathrm{d}$ show the measured (solid black lines) and fit (open circles) Stark signals at $\chi=90^{\circ}$ and $55^{\circ}$ respectively. Included with the fits are the contributions from the absorption spectra (red) and the first and second derivatives (dashed-dotted blue line and dashed green line respectively). 